\title{
Divergent Roles of miR-3162-3p in Pulmonary Inflammation in Normal and Asthmatic Mice as well as Antagonism of $m i R-3162-3 p$ in Asthma Treatment
}

\author{
Juman Liu ${ }^{\mathrm{a}}$ Yinhui Chen ${ }^{\mathrm{a}}$ Feng Zhang $^{\mathrm{b}} \mathrm{Xi}$ Peng $^{\mathrm{a}}$ Xiaoning Mao ${ }^{\mathrm{c}}$ \\ Weihong Lu ${ }^{d}$ Ruijian Wu ${ }^{a}$ Binglong Huang ${ }^{a}$ Yanmin Bao $^{e}$ Lian Ma ${ }^{f, g}$ \\ Yuge Huang $^{\text {a }}$ Xingliang Zhang ${ }^{\mathrm{f}, \mathrm{h}}$ \\ aDepartment of Pediatrics, The Affiliated Hospital of Guangdong Medical University, Zhanjiang, China; ${ }^{\text {bepartment }}$ \\ of Traditional Chinese Medicine, The Affiliated Hospital of Guangdong Medical University, Zhanjiang, China; \\ 'Clinical Laboratory, Shenzhen Children's Hospital, Shenzhen, China; dDepartment of Pediatrics, The First Affiliated \\ Hospital of Xinxiang Medical College, Weihui, China; 'Department of Respiratory Diseases, Shenzhen Children's

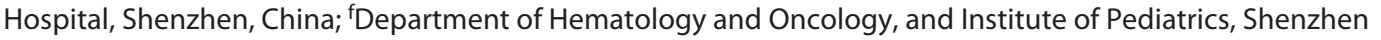 \\ Children's Hospital, Shenzhen, China; 9Department of Pediatrics, Second Affiliated Hospital of Shantou University \\ Medical College, Shantou, China; ' Department of Pediatric Surgery, Shenzhen Children's Hospital, Shenzhen, China
}

\section{Keywords}

Asthma $\cdot$ Mimic or antagomir $\cdot$ Inflammation $\cdot$ Animal models · Dexamethasone

\begin{abstract}
MicroRNA (miRNA) mimics or antagomirs hold great promise for asthma treatment compared with glucocorticoids as mainstay therapy for asthma. But the role of miRNA in regulating asthmatic inflammation is largely unclear. We previously reported that $m i R-3162-3 p$ in the peripheral blood of children with asthma was obviously upregulated compared to that in healthy children. This study aimed to elucidate the role of $m i R-3162-3 p$ in pulmonary inflammation in normal
\end{abstract}

Juman Liua, Yinhui Chena, and Feng Zhang contributed equally to this work.

Edited by: H.-U. Simon, Bern.

$\begin{aligned} & \text { karger@karger.com } \\ & \text { www.karger.com/iaa }\end{aligned}$
Karger $\%$

and asthmatic mice as well as preliminarily explore the potential of miR-3162-3p antagomir in asthma treatment. A noninvasive whole-body plethysmograph measured airway responsiveness. Both qRT-PCR and Western blot were used to detect the expression of miRNA, mRNA, or protein. Cells in bronchoalveolar lavage fluid were counted by platelet counting and Wright's staining. Inflammatory infiltration and mucus secretion were identified by hematoxylin and eosin and periodic acid-Schiff staining, respectively. Cytokines in the lungs were detected by ELISA. The miR-3162-3p mimic intraperitoneally administered to normal mice decreased $\beta$-catenin levels in the lungs without obviously altering the lung histology and cytokine levels. Antagonizing miR-3162-3p in ovalbumin-induced asthmatic mice effec-

Lian Ma

Institute of Pediatrics, Shenzhen Children's Hospital, Yitian Road 7019 Futian District, Shenzhen 518038 (China)

malian8965@sina.com

Yuge Huang

Department of Pediatics, The Affiliated Hospital of Guangdong Medical University Renmin Road No. 57, Zhanjiang 524001 (China)

Yug_Huang@163.com

Xingliang Zhang

Institute of Pediatrics, Shenzhen Children's Hospital, Yitian Road 7019

Futian District, Shenzhen 518038 (China)

xingliang_zhang@163.com 
tively alleviated the typical features of asthma, such as airway hyperresponsiveness, airway inflammation, and Th1/ Th2 cytokine imbalance, and concomitantly rescued the total and active $\beta$-catenin expression. Collectively, we discovered divergent roles of miR-3162-3p in lung inflammation between normal and asthmatic mice. The anti-inflammatory effects of the miR-3162-3p antagomir were comparable to those of glucocorticoid treatment. Our study helped in understanding the contribution of miRNAs to the pathogenesis of asthma.

(c) 2020 S. Karger AG, Basel

\section{Introduction}

Asthma, also known as bronchial asthma, is a common airway inflammatory disease. The incidence of asthma continues to rise globally. The onset of asthma will reduce the life quality of patients and increase the financial burden on their families $[1,2]$. Glucocorticoid-based treatment of asthma is effective, but long-term use harms children's development and growth.

MicroRNAs (miRNAs) are members of the noncoding RNA family, which can regulate gene expression at the posttranscriptional level $[3,4]$. There is growing evidence that miRNAs play a crucial role in the pathogenesis of asthma [5]. Many miRNAs are associated with asthmatic inflammation, showing either pro-inflammatory or antiinflammatory effects $[4,5]$, and may become a new strategy for asthma treatment by enhancing or inhibiting the expression of related miRNAs [6].

We have previously studied the difference in miRNA expression in peripheral blood between asthmatic and normal children by microarray and experimental evidence [7]. Luciferase assays and Western blot demonstrated that $\beta$-catenin was a new target of $m i R-3162-3 p$ due to a specific $m i R-3162-3 p$ pairing site in the $3^{\prime}$-UTR of $\beta$-catenin mRNA. The expression of $m i R-3162-3 p$ in the lungs of chicken egg ovalbumin (OVA)-induced asthmatic mice was significantly enhanced, whereas the mRNA and protein expression levels of the target gene $\beta$-catenin were decreased. MiR-3162-3p antagomir (anti$m i R-3162-3 p)$ treatment antagonized the endogenous $m i R-3162-3 p$ and effectively rescued the attenuation of endogenous $\beta$-catenin in OVA-induced asthmatic mice, as well as suppressed airway inflammatory infiltration in asthmatic mice [8].

In recent years, studies on the roles of miRNAs in the regulation of airway inflammation between normal and allergic mice have been limited. Moreover, whether a therapeutic intervention targeting miRNA is as effective as glucocorticoid treatment is unclear. In this study, the chemically synthesized analog $m i R-3162-3 p$ mimic (mimic-miR-3162-3p) was transfected intraperitoneally into normal mice to investigate the effects of $m i R-3162-3 p$ on the expression of $\beta$-catenin and inflammatory response in the lung tissue. Dexamethasone (DXM) or anti$m i R-3162-3 p$ was injected into asthmatic mice to assess the therapeutic effect of anti-miR-3162-3p on pulmonary inflammation. Comparing the different impacts of mimic-miR-3162-3p on pulmonary inflammation in normal mice and anti-miR-3162-3p in asthmatic mice may be useful in further understanding the role of $m i R-3162-3 p$ in the pathogenesis of asthma, and provide the experimental basis for the clinical application of miRNA inhibitors in asthma treatment.

\section{Materials and Methods}

Animals

Female Balb/c mice (6-8 weeks old, $20 \pm 2$ g) were obtained from the Laboratory Animal Center of Guangdong Medical University. All procedures of animal experiments were approved by the Laboratory Animal Center of Guangdong Medical College Animal Care and Use Committee and conform to the Guide for the Care and Use of Laboratory Animals.

\section{Treatment of Normal Mice with Mimic-miR-3162-3p}

The oligonucleotides of mimic-miR-3162-3p, chemically synthesized and specially modified with $2^{\prime}$-ome and their ends with $5^{\prime}$-cholesterol for animal transfection by Biomics Biotechnologies Co., Ltd. (Suzhou, China), were dissolved in endotoxin-free water, and the working dilution was prepared in animals RNA transfection reagent Entranster ${ }^{\mathrm{TM}}$-in vivo (18668-11-1; Engreen Biosystem Co., Ltd.) [9]. Then, 2, 4, or $8 \mathrm{mg} / \mathrm{kg}$ of mimic- $m i R-3162-3 p$ oligonucleotides in $0.4 \mathrm{~mL}$ was intraperitoneally delivered into the normal mice for 3 consecutive days. The mimic-miR-3162-3p solution was replaced by sterile saline in the normal control (NC) group. All oligonucleotide sequences are specified as follows: mimic-miR3162-3p (mimic): F $5^{\prime}$-UCCCUACCCCUCCACUCCCCA-3', R 5'-UGGGGAGUGGAGGGGUAGGGA-3'. Each group contained 18 mice.

Treatment of Asthmatic Mice with Anti-miR-3162-3p or DXM The OVA (A5503; Sigma)-induced asthma model of mice was successfully established as described previously [8, 10] (Table 1; Fig. 4a). Mice were divided into 4 groups according to sensitization/treatment/challenge, including the NC group (saline/saline/ saline), asthmatic group (OVA/saline/OVA), antagomir group (OVA/anti-miR-3162-3p/OVA), and DXM group (OVA/DXM/ OVA). Then, $4 \mathrm{mg} / \mathrm{kg}$ anti-miR-3162-3p in $0.4 \mathrm{~mL}$ of endotoxinfree water + animal RNA transfection reagent or $2 \mathrm{mg} / \mathrm{kg} D X M$ in $0.4 \mathrm{~mL}$ of endotoxin-free water was intraperitoneally administered. MiR-3162-3p inhibitor (antagomir): 5'-UGGGGAGUGGAGGGGUAGGGA-3'. The synthesis and usage of anti-miR- 
Table 1. Groups and treatment of mice in the experiments ( $n=18$ each group)

\begin{tabular}{|c|c|c|c|}
\hline Group & $\begin{array}{l}\text { Sensitization } \\
\text { d0, d7, d14 }\end{array}$ & $\begin{array}{l}\text { Treatment } \\
\mathrm{d} 20, \mathrm{~d} 21, \mathrm{~d} 22, \mathrm{~d} 23\end{array}$ & $\begin{array}{l}\text { Challenge ( } 30 \mathrm{~min} \text { ) } \\
\mathrm{d} 21, \mathrm{~d} 22, \mathrm{~d} 23\end{array}$ \\
\hline NC & Saline $0.2 \mathrm{~mL}$ & Endotoxin-free water $0.4 \mathrm{~mL}$ & Saline $5 \mathrm{~mL}$ \\
\hline Asthma & $\mathrm{OVA}^{\mathrm{a}}$ & Endotoxin-free water $0.4 \mathrm{~mL}$ & $1 \% \mathrm{OVA}^{\mathrm{d}}$ \\
\hline Antagomir & $\mathrm{OVA}^{\mathrm{a}}$ & Antagomir ${ }^{\mathrm{b}}$ & $1 \% \mathrm{OVA}^{\mathrm{d}}$ \\
\hline DXM & $\mathrm{OVA}^{\mathrm{a}}$ & $\mathrm{DXM}^{\mathrm{c}}$ & $1 \% \mathrm{OVA}^{\mathrm{d}}$ \\
\hline
\end{tabular}

OVA, ovalbumin; DXM, dexamethasone; NC, normal control. ${ }^{\text {a }}$ Sensitization by intraperitoneal injection of $50 \mu \mathrm{g}$ OVA emulsified with $2 \mathrm{mg}$ aluminum hydroxide $\left(23918\right.$, Sigma) in $0.2 \mathrm{~mL}$ saline. ${ }^{\mathrm{b}}$ Treatment with $4 \mathrm{mg} /$ $\mathrm{kg}$ miR-3162-3p antagomir in $0.4 \mathrm{~mL}$ of endotoxin-free water + animal RNA transfection reagent. ${ }^{\mathrm{c}}$ Treatment with $2 \mathrm{mg} / \mathrm{kg}$ DXM in $0.4 \mathrm{~mL}$ of endotoxin-free water. ${ }^{\mathrm{d}}$ Challenge with $1 \%$ OVA in $5 \mathrm{~mL}$ saline using a nebulizer.

3162-3p were similar to those of mimic-miR-3162-3p. The oligonucleotide bases of mimic, antagomir, and controls were modified with $2^{\prime}$-ome, and their ends with $5^{\prime}$-cholesterol. The control of mimic- $m i R-3162-3 p$ or anti-miR-3162-3p, consisting of a scrambled oligonucleotide, was rationally omitted because the specificity of mimic-miR-3162-3p or anti-miR-3162-3p had been proved in our previous publication [8].

Analysis of miRNAs and mRNA Expression by $q R T-P C R$

Total RNA was isolated with RNAiso Plus (9109; Takara), and miRNAs were extracted using the miRcute miRNA kit (DP501), followed by a reverse transcription reaction. The qRT-PCR for miRNA and mRNA was performed with the SYBR PrimeScript miRNA RT-PCR kit (RR716; TaKaRa) and PrimeScript RT reagent kit with gDNA Eraser (RR047A), respectively, on a ROCHE LightCycler 480 II real-time PCR system. The $2^{-\Delta \Delta \mathrm{Ct}}$ method was used to analyze the relative changes of the target gene to the endogenous control. The qPCR primers were purchased from Sangon (Shanghai, China), including miR-3162-3p: F $5^{\prime}$-UCCCUACCCCUCCACUCCCCA-3', R 5'-CTACCCCTCCACTCCCCAAAA-3'; F_RNU6B: 5' -CTCGCTTCGGCAGCACA-3', R_ RNU6B: $5^{\prime}$-AACGCTTCACGAATTTGCGT- $3^{\prime} ; \quad$ F_ $\beta$-catenin: $5^{\prime}$-TGGTGACAGGGAAGACATCA-3'，R_ $\beta$-catenin: $5^{\prime}$-CCATAGTGAAGGCGAACTGC- $3^{\prime} ; \quad$ F_ $\beta$-actin: $5^{\prime}$-TCCGTAAAGACCTCTATGCCAACA- $3^{\prime}$, R_ $\beta$-actin: $5^{\prime}$-GCTAGGAGCCAGAGCAGTAATCTC- $3^{\prime}$. Three independent experiments were performed in triplicate.

\section{Respiratory Responses to Methacholine}

Based on our previous procedures $[8,10]$, the enhanced pause (Penh) value of unrestrained mice inhaling different doses of methacholine aerosol was measured by a noninvasive whole-body plethysmograph (Buxco, NY, USA).

\section{Histological Examination}

Based on our published method $[8,10,11]$, the left lung sections were stained with hematoxylin and eosin (C0105; Beyotime, Shanghai, China) and periodic acid-Schiff (PAS) (G1280; Solarbio, Beijing, China). Based on the published method $[10,12]$, the blinded histopathological score was used to assess the degree of peribronchial and alveolar structural changes on a subjective scale of
0-3: 0, airway epithelial cells remain intact; 1 , lung parenchyma becomes dense, alveolar cavity becomes narrowed; 2, columnar cells become damaged; and 3, airway wall is destroyed with visible effusion. Six mice were chosen from each group, and 3 visual fields of 1 lung section were selected from each mouse for histological scoring. Total lung structural change was defined as the average of the peribronchial and alveolar structural impairment scores.

\section{Inflammatory Cells from Bronchoalveolar Lavage Fluid}

According to our published procedures $[8,10]$, collection of BALF and cell counts were performed by using a hemocytometer and Wright staining blindly.

\section{Biochemical Detections}

The protein expression levels were detected by Western blot as described previously [11]. Total $\beta$-catenin rabbit antibody $(1: 2,000$, \#8480, Cell Signaling Technology [CST]), non-phospho (active) $\beta$-catenin rabbit antibody $(1: 2,000, \# 8814 \mathrm{~S}, \mathrm{CST}), \beta$-actin rabbit antibody (1:3,000, \#4967S, CST), and horseradish peroxidase-conjugated anti-rabbit IgG $(1: 2,000, \# 7074$, CST) were used for antigen-antibody reaction. Immobilon Western Chemiluminescent HRP Substrate (Millipore, WBLUR0500) was used for visualization of immunoreactive bands, and densitometric analysis was conducted using the image software ImageJ.

Production of tumor necrosis factor- $\alpha$ (TNF- $\alpha)(B M S 607 / 3$; BioSource), interleukin-2 (IL-2) (KMC0021; Invitrogen), IL-6 (KMC0061; Invitrogen), interferon- $\gamma$ (IFN- $\gamma$ ) (BMS606; BioSource), IL-4 (EMIL4; Invitrogen), IL-5 (PI620; Beyotime), IL-10 (BMS614/2; BioSource), IL-13 (P5948; Beyotime), IL-17A (EMIL17A; Invitrogen), and IL-23 (BMS6017; BioSource) in the lung tissue supernatant was measured by ELISA according to the manufacturer's instructions.

\section{Statistical Analysis}

Data were analyzed with GraphPad Prism 7.0 and expressed as mean \pm SD. Comparisons among groups were performed with one-way ANOVA, followed by Tukey's multiple comparison post hoc test. For analysis of heterogeneity of variance, the $K$ independent samples nonparametric test and Kruskal-Wallis test were used. Each experiment was repeated independently 3 times, and the criterion for statistical significance was $p \leq 0.05$. 


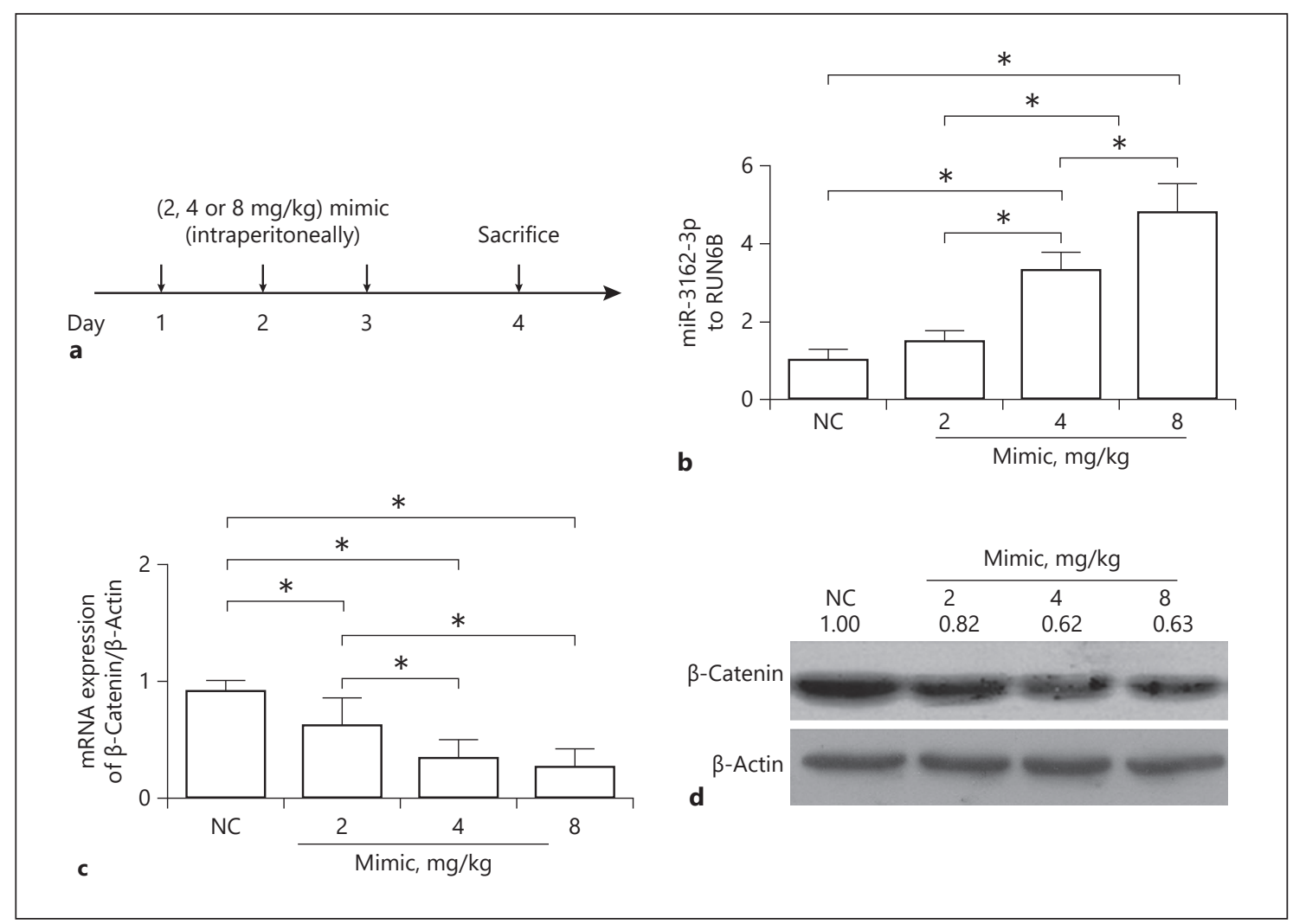

Fig. 1. Expression level of $\beta$-catenin in the lungs after intraperitoneal administration of mimic-miR-3162-3p. a Mice were injected with 2,4 , or $8 \mathrm{mg} / \mathrm{kg}$ mimic according to their weight into the abdominal cavity on days 1 , 2 , and 3, and sacrificed on day 4. b, c Expression levels of $m i R-3162-3 p$ and $\beta$-catenin mRNA in the lungs detected by qRT-PCR. $\mathbf{d}$ Expression of $\beta$-catenin protein in the lungs detected by Western blot. The average relative quantitative densitometric values of total $\beta$-catenin protein were added on the top of the protein band. ${ }^{*} p<0.05$. NC, normal control.

\section{Results}

\section{$\beta$-Catenin mRNA and Protein Expression Levels} in Normal Mice upon Intraperitoneal Injections of Mimic-miR-3162-3p

The mimic-miR-3162-3p was intraperitoneally injected into mice to study the effects of $m i R-3162-3 p$ on the lungs of normal mice (Fig. 1a). Compared with the NC group, the total $m i R-3162-3 p$ (including transfected mimic and endogenous $m i R-3162-3 p$ ) in thelungs showed an ascent tendency as the dose of mimic increased. The total $m i R-3162-3 p$ increased significantly in a dose-dependent manner after injection with mimic at 4 or $8 \mathrm{mg} /$ $\mathrm{kg}$ (Fig. 1b). The mRNA level of $\beta$-catenin had an opposite trend of the mimic-miR-3162-3p dose. The mRNA level of $\beta$-catenin upon 3 doses of mimic administration showed significant downregulation compared to that in the NC group; 4 and $8 \mathrm{mg} / \mathrm{kg}$ mimic exerted more inhib- itory capacity than $2 \mathrm{mg} / \mathrm{kg}$ mimic (Fig. 1c). In comparison with the NC group, administration with 2 and $4 \mathrm{mg} /$ $\mathrm{kg}$ mimic-miR-3162-3p significantly decreased the expression of the $\beta$-catenin protein, but $8 \mathrm{mg} / \mathrm{kg}$ did not continue to reduce $\beta$-catenin protein (Fig. 1d, online suppl. Fig. S1A; for all online suppl. material, see www. karger.com/doi/10.1159/000507250).

\section{Inflammatory Filtration and Mucus Secretion in the Lungs of Normal Mice upon Mimic-miR-3162-3p Administration}

To evaluate the effects of $m i R-3162-3 p$ on the inflammation of normal mice lungs, total cells and differential cell counts in the bronchoalveolar fluid (BALF) were statistically analyzed after intraperitoneal transfection of mimic-miR-3162-3p. With the increase of the mimic dose, the number of total cells showed an upward trend, but there was no significant difference (Fig. 2a). Macro- 

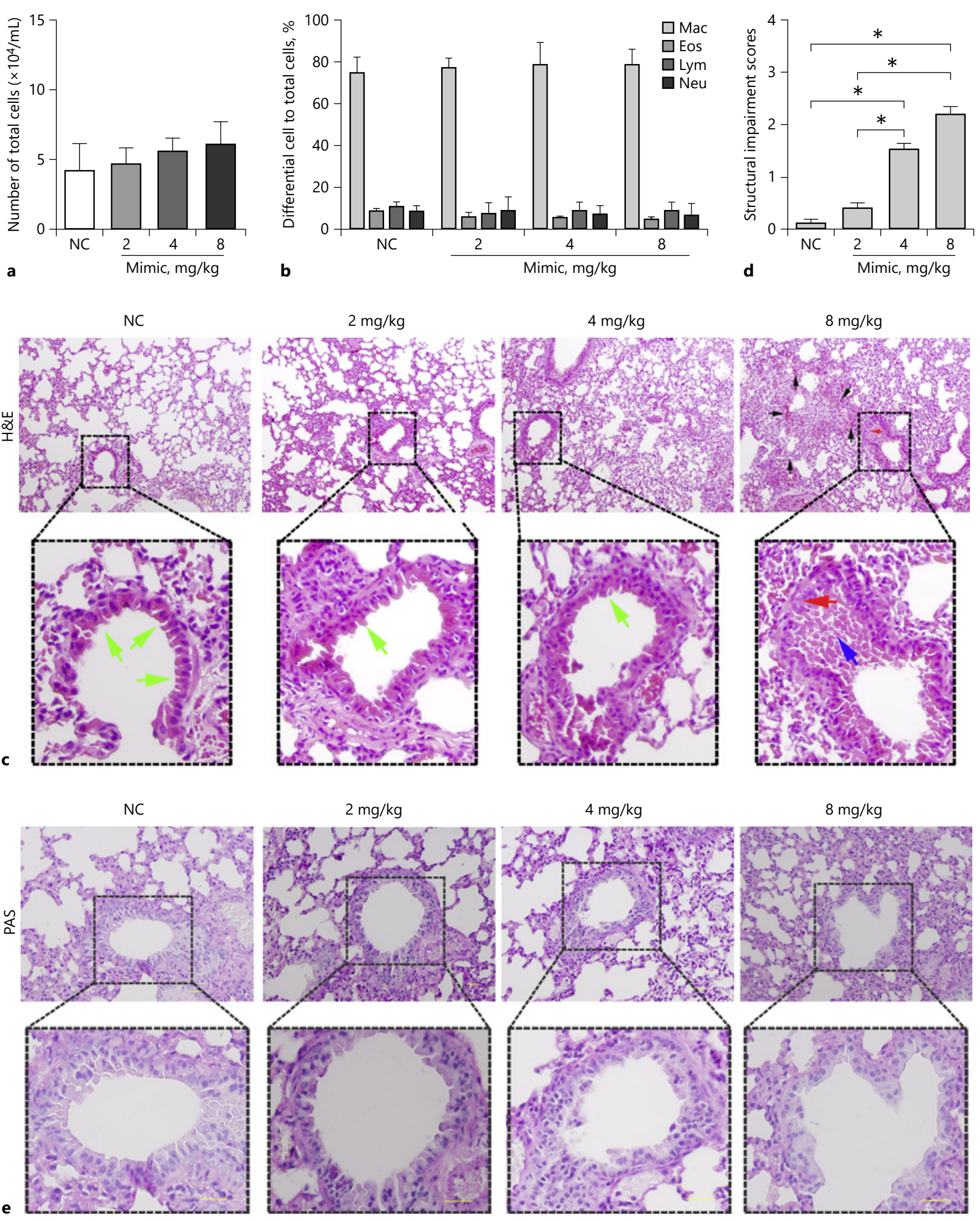

2

(For legend see next page.)

Int Arch Allergy Immunol 2020;181:594-605 DOI: 10.1159/000507250
Liu/Chen/Zhang/Peng/Mao/Lu/Wu/ Huang/Bao/Ma/Huang/Zhang 
phages were predominant, whereas other differential cell proportions were very low in BALF among the 4 groups. The profiles of differential cell counts in BALF were not obviously altered in response to different doses of the mimic (Fig. 2b).

The mimic had no significant effect on eosinophil infiltration in both perivascular and peribronchial regions (Fig. 2c). Nevertheless, with the increase of the mimic dose, the lung parenchyma became dense, the alveolar septum widened, the alveolar cavity narrowed, and columnar epithelial cells of the airway were destroyed with clearly visible deformations of the airway cross-section and destruction of ciliary columnar cells (Fig. 2c, d). No obvious mucus hypersecretion was found in any group (Fig. 2e).

\section{Patterns of Cytokines in Lung Homogenate upon}

Mimic-miR-3162-3p Administration

Compared with the NC group, TNF- $\alpha$ did not change significantly in the $2-\mathrm{mg} / \mathrm{kg}$ group but increased significantly in the $4-$ and $8-\mathrm{mg} / \mathrm{kg}$ groups. With the increase of mimic-miR-3162-3p, the concentration of IL- 6 increased in a dose-dependent manner, and there was a significant increase in the $4-$ and $8-\mathrm{mg} / \mathrm{kg}$ groups in comparison to the NC group. IL-2, IFN- $\gamma$, IL-4, and IL-10 did not change conspicuously (Fig. 3).

\section{Effects of Anti-miR-3162-3p and DXM on $\beta$-Catenin} Expressions in Asthmatic Mice Lungs

An OVA-induced asthmatic mice model was established (Fig. 4a, b). To study the effect of antagonizing $m i R-3162-3 p$ on airway hyperresponsiveness, mice were pretreated with anti-miR-3162-3p or DXM. The en-

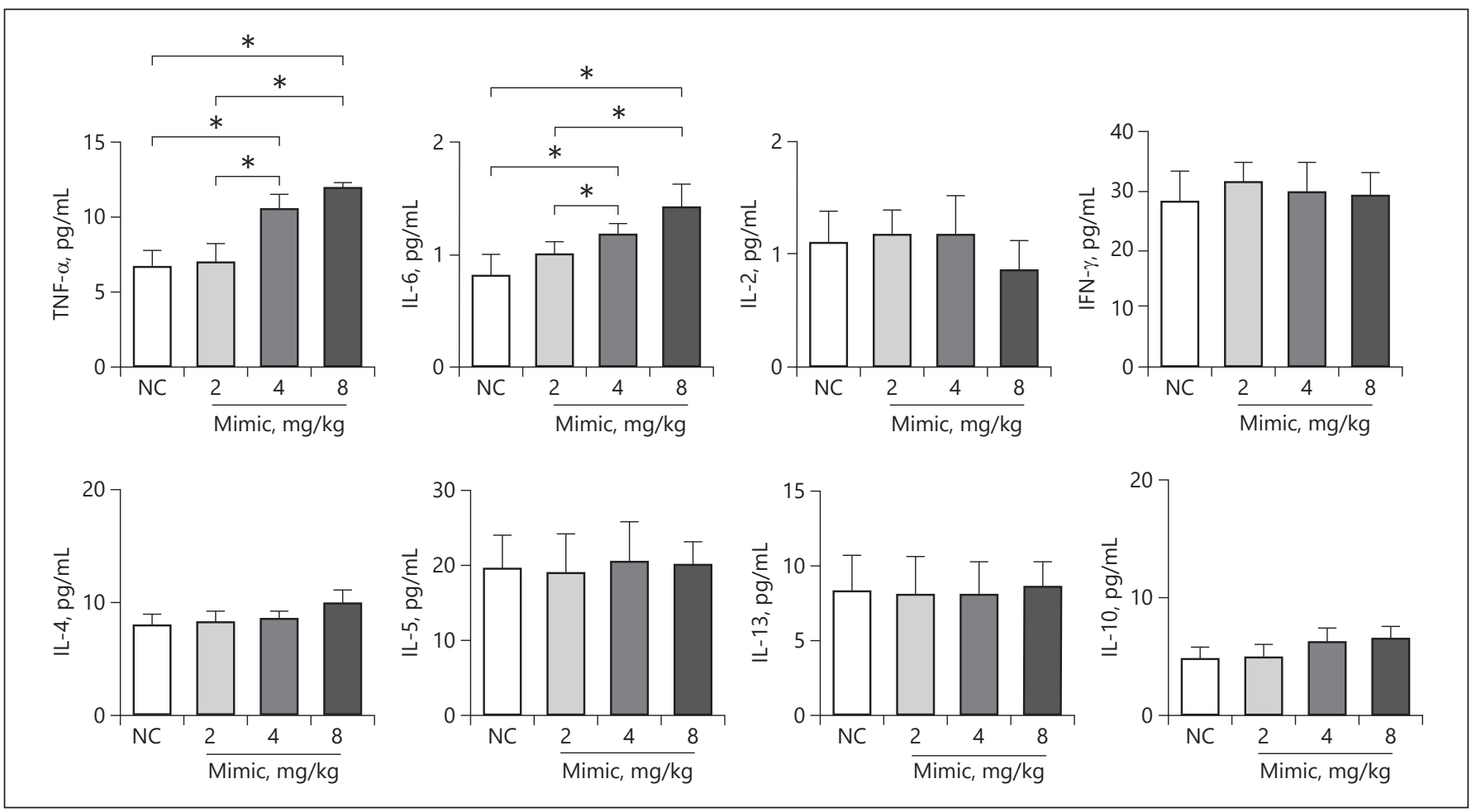

Fig. 3. Levels of cytokines in lung homogenates were detected by ELISA. ${ }^{*} p<0.05$. TNF- $\alpha$, tumor necrosis factor- $\alpha$; IL, interleukin; IFN- $\gamma$, interferon- $\gamma$.

Fig. 2. Recruitment of inflammatory cells to the lung tissue and induction of mucous cell metaplasia in response to mimic-miR-3162-3p. a, b Total cells and the proportion of differential cells in BALF. c, d Representative lung sections by H\&E staining. Osmotic hemorrhage (black arrows), columnar epithelial cells (green arrows), damaged airway wall (red arrow), and effusion on the airway (blue arrows). ${ }^{*} p<0.05$. e Representative airway sections by PAS staining. Magnification, $\times 100$; scale bar, $50 \mathrm{~nm}$. PAS, periodic acid-Schiff; H\&E, hematoxylin and eosin; NC, normal control; Leu, leucocytes; Eos, eosinophils; Lym, lymphocytes; Neu, neutrophils.

Roles of $m i R-3162-3 p$ in Inflammation and Asthma Treatment
Int Arch Allergy Immunol 2020;181:594-605 DOI: $10.1159 / 000507250$ 


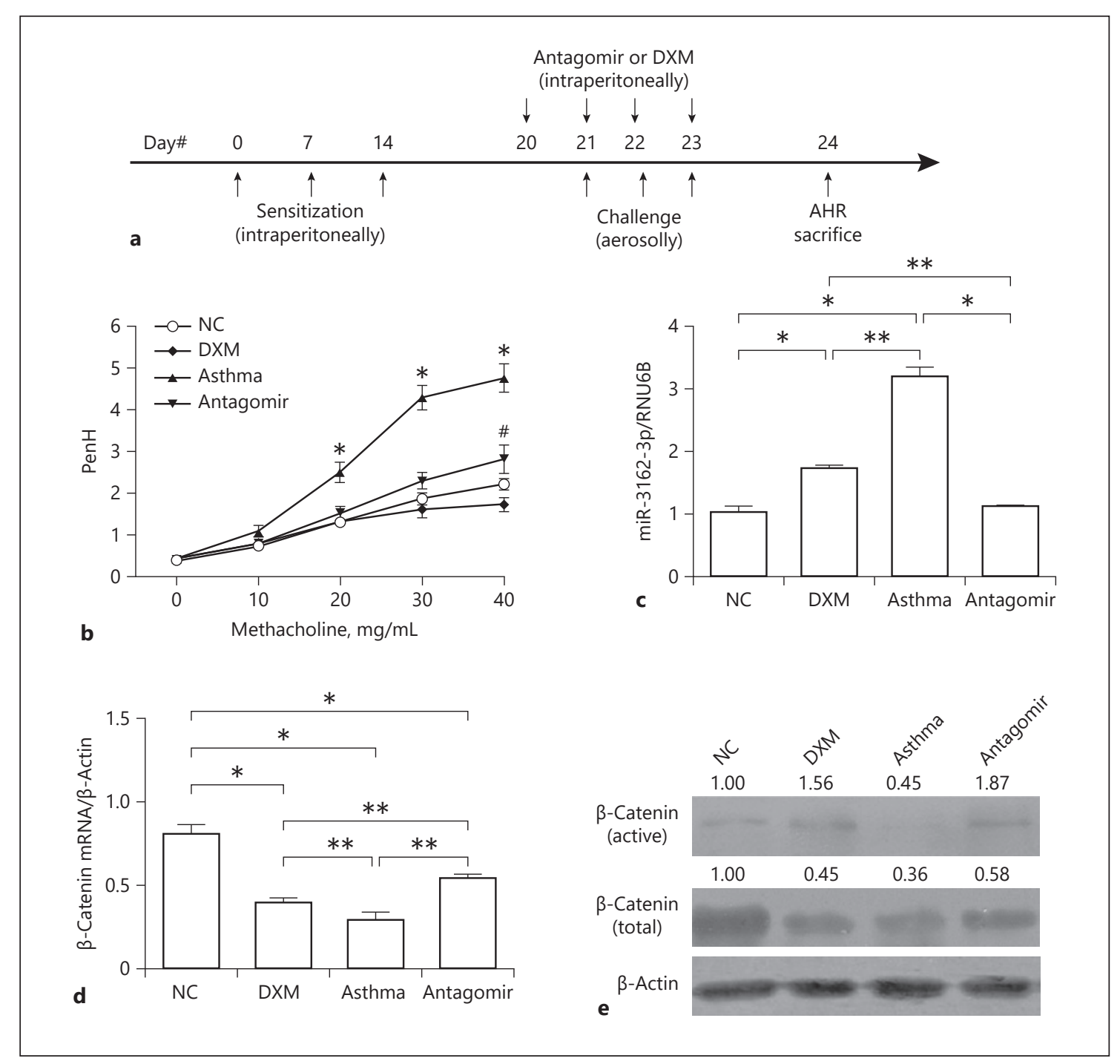

Fig. 4. Effects of anti-miR-3162-3p or DXM on airway hyperresponsiveness and $\beta$-catenin expressions in the lungs of asthmatic mice. a Schematic representation of anti-miR-3162-3p or DXM administration in OVA-induced asthmatic mice. b Airway responsiveness under increasing concentrations of methacholine. ${ }^{*} p<0.05$ versus NC, antagomir, or DXM, ${ }^{\#} p<0.05$ versus DXM. c, d Contents of $m i R-3162-3 p$ and $\beta$-catenin in the lungs were detected by qRT-PCR. ${ }^{*} p<0.05,{ }^{* *} p<0.001$. e Active and total $\beta$-catenin proteins in the lungs were detected by Western blot. The average relative quantitative densitometric values of $\beta$-catenin proteins were added to the top of the protein band. OVA, ovalbumin; DXM, dexamethasone; AHR, airway hyperreactivity; NC, normal control.

hanced pause (Penh) value decreased significantly with 20 , 30, and $40 \mathrm{mg} / \mathrm{mL}$ methacholine. There were no evident differences in airway hyperresponsiveness between anti-miR-3162-3p and DXM treatment in response to methacholine at 10, 20, and $30 \mathrm{mg} / \mathrm{kg}$ (Fig. 4b).

The qRT-PCR results showed that anti-miR-3162-3p could significantly reduce the endogenous $m i R-3162-3 p$ in the lungs of asthmatic mice, which are close to the NC group. DXM could reduce the endogenous $m i R-3162-3 p$ in the lungs of asthmatic mice by about half, which was conspicuously not as effective as that of anti-miR-3162-3p (Fig. 4c). The mRNA expression of $\beta$-catenin in asthmatic mice was significantly lower than that in the NC group. The inhibition of $\beta$-catenin mRNA expression was dramatically relieved in the antagomir group, which was noticeably more effective than that in the DXM group (Fig. 4d). Similarly, both anti-miR-3162-3p and DXM partially rescued the reduced total $\beta$-catenin protein in 


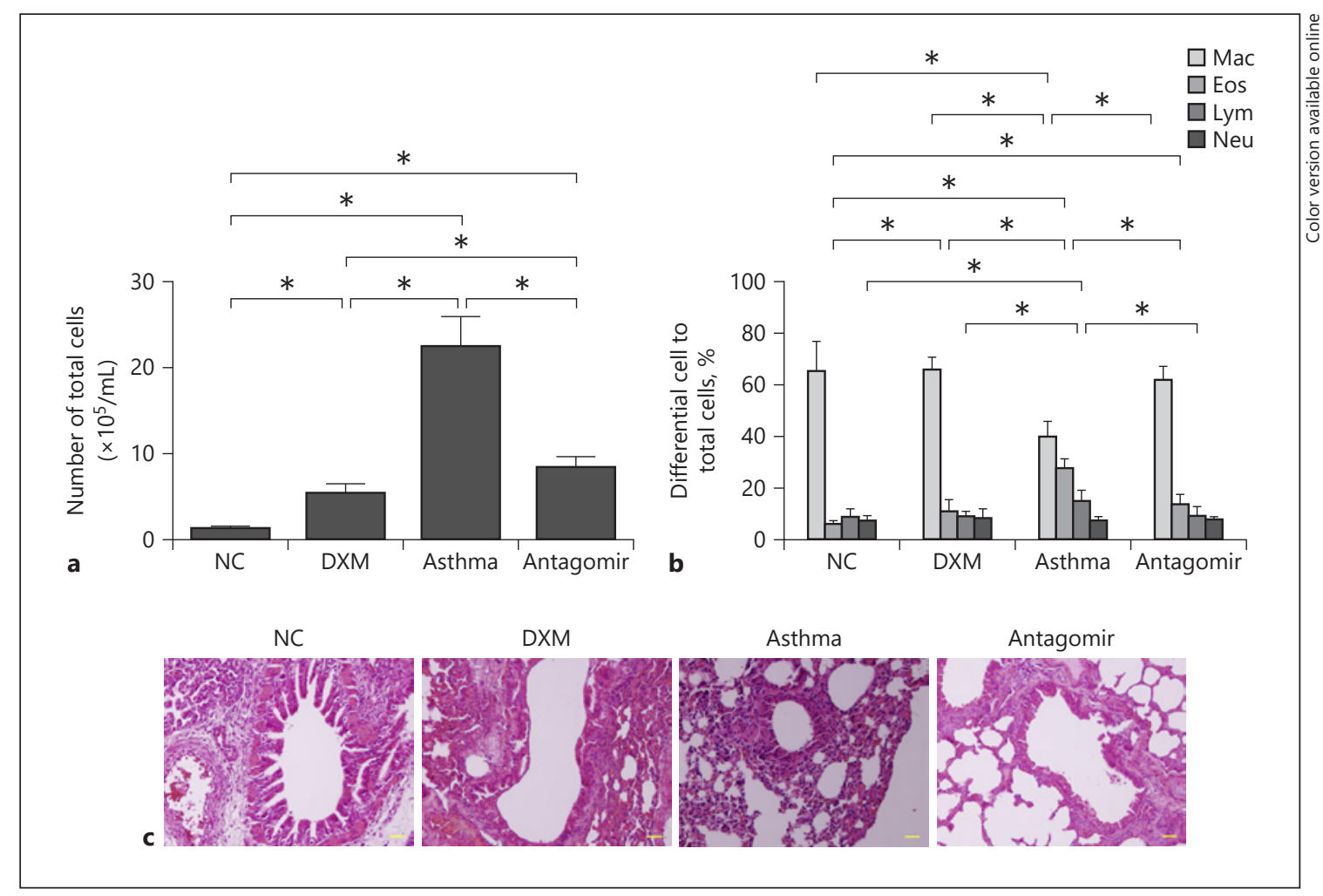

Fig. 5. Recruitment of inflammatory cells to the lungs. a Total cells in BALF. ${ }^{*} p<0.05$. b Proportions of differential cells in BALF. ${ }^{*} p<0.05$. c Representative lung sections were H\&E stained. Magnification, $\times 200$; scale bar, $50 \mathrm{~nm}$. H\&E, hematoxylin and eosin; Leu, leucocytes; Eos, eosinophils; Lym, lymphocytes; Neu, neutrophils; DXM, dexamethasone; NC, normal control.

the asthmatic group. Of note, anti-miR-3162-3p and DXM could near-fully reverse or even increase the active $\beta$-catenin protein compared to the counterpart in the NC group (Fig. 4e, online suppl. Fig. S1B, C).

\section{Inflammatory Filtration in the Lungs of}

Asthmatic Mice upon Anti-miR-3162-3p and DXM

\section{Administration}

The number of total cells and the proportion of eosinophils and lymphocytes in BALF of OVA-induced asthmatic mice increased significantly (Fig. 5a, b). After treatment with anti-miR-3162-3p or DXM, the number of total cells and the proportion of eosinophils decreased significantly, but these were still more than that in the counterparts in the NC group. The proportion of macrophages increased significantly, whereas the proportion of lymphocytes declined adjacent to that in the $\mathrm{NC}$ group when the asthmatic mice were injected with anti-miR-3162-3p or DXM. The inhibitory effect of anti-miR-3162-3p on BALF total cells in asthmatic mice was less than that of DXM, but there was a similar pattern of differential cell counts in BALF between these 2 groups.

The inflammatory infiltration, mainly EOS, around the bronchi and vessels increased significantly in the asthmatic group compared to those in the NC group. The structural disorder of lung parenchyma aggravated, and columnar epithelial cells of the airway were significantly damaged. After treatment with anti-miR-3162-3p or DXM, the inflammatory infiltration around the bronchi in asthmatic mice decreased significantly. The structure of airway epithelial cells in the antagomir group remained relatively intact, but the structure of airway epithelial cells in the DXM group was obviously damaged (Fig. 5c).

\section{Cytokine Pattern in the Lung Homogenate upon}

Anti-miR-3162-3p or DXM Administration

Many cytokines are involved in the immuno-inflammatory mechanism of asthma. Compared with the NC group, the expression levels of TNF- $\alpha$ and IL- 6 in asth- 

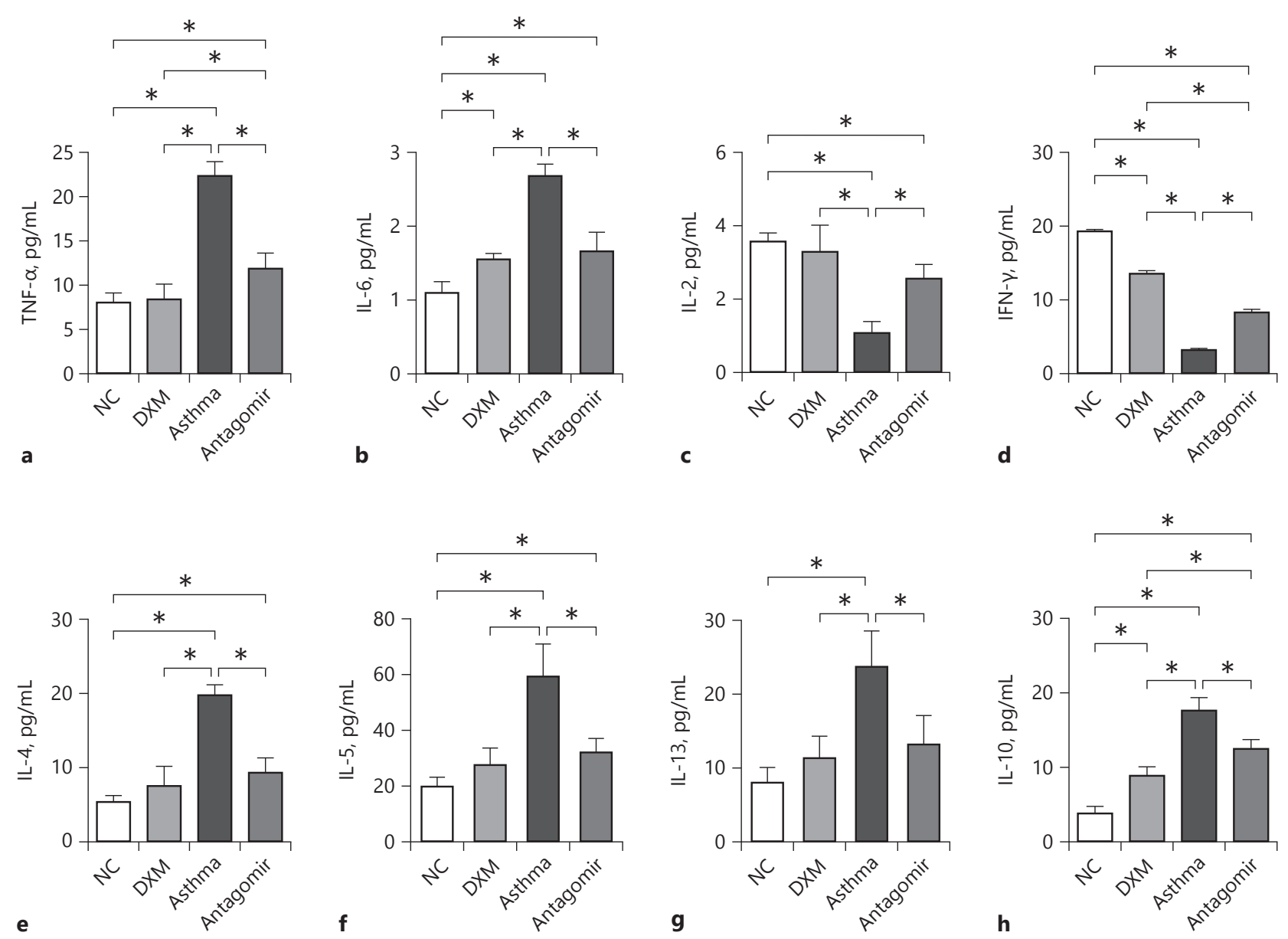

Fig. 6. Levels of cytokines in lung homogenates upon anti-miR-3162-3p or DXM administration. a TNF- $\alpha$. b IL6. c IL-2. d IFN- $\gamma$. e IL-4. f IL-5. g IL-13. h IL-10. $N=6-8 .{ }^{*} p<0.05$. TNF- $\alpha$, tumor necrosis factor- $\alpha$; IL, interleukin; IFN- $\gamma$, interferon- $\gamma$; DXM, dexamethasone; NC, normal control.

matic mice were significantly increased. The expression of TNF- $\alpha$ in asthmatic mice generally decreased after treatment with anti-miR-3162-3p and decreased more after DXM administration, which approximated the level of the NC group (Fig. 6a). The inhibitory effects of anti$m i R-3162-3 p$ and DXM on the expression of IL-6 in the lungs were similar. Both treatments could decrease the level of IL-6 of asthmatic mice, but not to the equivalent level of the NC group (Fig. 6b). The expression of Th1 cytokines such as IL- 2 and IFN- $\gamma$ in the asthmatic group decreased significantly. DXM could reverse the expression of IL-2 close to that in the NC group, showing more potent capacity than anti-miR-3162-3p. Both anti-miR3162-3p and DXM were relatively weak for restoring the expression of IFN- $\gamma$ in asthmatic mice. The restoring effect of anti-miR-3162-3p on IFN- $\gamma$ expression was far less than that of DXM (Fig. 6c, d). The expression level of Th2 cytokines such as IL-4, IL-5, IL-10, and IL-13 in asthmatic mice was upregulated. After treatment with anti-miR$3162-3 p$ or DXM, the expression levels of these Th2 cytokines were significantly decreased. DXM could reduce the expression level of IL-4, IL-5, and IL-13 close to the NC group, but the inhibitory effect of anti-miR-3162-3p on IL-4, IL-10, and IL-13 could not reach the same level as the NC group (Fig. 6e-h). IL-17A and IL-23 produced by Th17 cells were not significantly changed among the 4 groups (online suppl. Fig. S2). 


\section{Discussion}

MiRNAs play an important role in airway allergic inflammation $[4,5,13]$. We investigated $m i R-3162-3 p$ in peripheral blood as a potential diagnostic biomarker to discriminate childhood asthma patients from healthy subjects [7]. We further confirmed that anti-miR-3162-3p could effectively reduce eosinophil infiltration around the airway of OVA-induced asthmatic mice [8]. Allergic inflammation is typically characterized by a Th2 cell cytokine-like response, with upregulation of Th2 (e.g., IL-4, IL-5, and IL-13) and downregulation of Th1 (e.g., IL-2 and IFN- $\gamma$ ) cytokines $[4,14]$. In this study, anti-miR$3162-3 p$ could increase IL- 2 and IFN- $\gamma$, and decrease IL4 , IL-5, and IL-13 in the lungs of asthmatic mice, which is similar to anti-mmu-mir-106a in OVA-induced asthmatic mice [15]. After the knockout of mmu-mir-106a, the expression level of IL-10 in the lungs increased, which could improve airway inflammation [15]. Nevertheless, the expression of IL-10 in the lungs of asthmatic mice was reduced by anti-miR-3162-3p, and concomitant with the ameliorated airway hyperresponsiveness. Besides, the normal mice transfected with mimic-miR-3162-3p showed an upward trend of total cells in BALF, but there was no significant change in BALF cells and inflammatory infiltration. Similarly, nebulization of $m m u-m i R-$ $106 a$ to normal mice did not alter differential cell or lung histology [15]. Both TNF- $\alpha$ and IL-6 are pro-inflammatory cytokines that mediate essential functions. There was a dose-dependent increase in TNF- $\alpha$ levels in mouse macrophage cell line RAW 264.7 cells with $m m u-m i R$ $106 a$ transfection [15]. The miR-570-3p transfection of airway epithelial cells increases IL-6 expression [12]. Intriguingly, the expression of IL- 6 or TNF- $\alpha$ in the lungs of normal mice was increased by the mimic-miR-3162-3p. The knockdown of $\beta$-catenin by its siRNA significantly increased IL-6 expression in LPS-treated RAW 264.7 cells [16]. Therefore, we postulated that the upregulation of IL-6 level might be achieved by the knockdown of $\beta$-catenin by mimic-mir-3162-3p. Nevertheless, several Th1 cytokines (IL- 2 and IFN- $\gamma$ ) and Th2 cytokines (IL-4, IL-5, IL-10, and IL-13) were not noticeably altered. Based on the difference in lung inflammations between normal mice in response to mimic-miR-3162-3p and asthmatic mice in response to anti-miR-3162-3p, a preliminary conclusion could be drawn that the high expression of endogenous $m i R-3162-3 p$ in the lungs of OVA-induced asthmatic mice may mainly change the balance of Th1/Th2 cytokines by influencing sensitization and challenge-mediated inflammatory response.

Roles of $m i R-3162-3 p$ in Inflammation and Asthma Treatment
Different sensitization methods produce diverse patterns of inflammatory infiltration and cytokines [17], which may have an essential correlation with a $\beta$-catenin signal [18]. Intranasal sensitization and chronic challenge caused neutrophilic infiltration and high levels of IL-17 in the lungs for activation of $\beta$-catenin signaling in house dust mite-induced asthmatic mice [19]. In toluene di-isocyanate-induced asthma, higher amounts of neutrophils and eosinophils in BALF were found, and the total $\beta$-catenin, as well as the phospho- $\beta$-catenin (Tyr654) protein content in the lungs, increased [20]. The increased expression of miRNA-943-3p in the lungs of OVA-induced asthmatic mice increased activated $\beta$-catenin but not total $\beta$-catenin protein. Administration of miRNA-943-3p antagomir could effectively reverse the activation of $\mathrm{Wnt} / \beta$-catenin and alleviate airway inflammation in mice [19]. However, several reports showed that the expression of $\beta$-catenin in asthmatic patients or asthmatic mice was downregulated $[21,22]$. The high expression of $m i R-3162-3 p$ in the lungs also could downregulate $\beta$-catenin protein and aggravate the eosinophil infiltration in the lungs of OVA-induced asthmatic mice [8]. Here, anti-miR-3162-3p could effectively retain the active $\beta$-catenin protein level and reverse the imbalance of Th1/Th2 immune cytokines in the lungs of asthmatic mice. Therefore, it is speculated that $m i R$ 3162-3p may affect the inflammatory pattern of neutrophil or eosinophil-neutrophil mixed asthma by interfering with the $\beta$-catenin signaling.

Glucocorticoids are used as mainstay therapy for asthma, but some patients remain resistant to treatment, or long-term drug control has some serious side effects. Therefore, exploring and developing highly effective and individualized new drugs to treat asthma is of great significance. MiRNA agonists or antagonists hold the promise as biomarkers and targets of therapy in asthma $[23,24]$. Inhibition of miRNA-145 by antagomir can reduce eosinophilic inflammation, mucus hypersecretion, Th2 cytokine production, and airway hyperresponsiveness, which is comparable to glucocorticoid treatment [25]. Similarly, anti-miR-3162-3p could effectively reduce airway hyperresponsiveness and pulmonary inflammation in asthmatic mice, close to the therapeutic effects of DXM. Although many basic studies have shown that miRNAs displayed good effects on asthma treatment, their nonspecific targets and side effects need further investigations [5]. In this study, when normal mice were treated with a high dose of mimic-miR-3162-3p, the columnar epithelial cells of the airway were damaged with bleeding and leakage.

In animal models of asthma, some drugs targeting the $\mathrm{Wnt} / \beta$-catenin signaling pathway can improve asthmatic

Int Arch Allergy Immunol 2020;181:594-605 603 
inflammation and airway remodeling. The $\beta$-catenin inhibitors XAV-939 and ICG-001 can significantly reverse airway inflammation and airway hyperresponsiveness in toluene di-isocyanate-induced asthmatic mice [26]. In this study, both anti-miR-3162-3p and DXM effectively inhibited the expression of $\beta$-catenin in the lungs of OVA-induced asthmatic mice. Therefore, it is speculated that both anti-miR-3162-3p and DXM may regulate lung inflammation and airway hyperresponsiveness through the $\beta$-catenin signaling pathways. There are complicated regulatory networks between the $\beta$-catenin signaling pathway and other signaling pathways. Except for $\beta$-catenin, other target proteins of $m i R-3162-3 p$ involved in the pathogenesis of asthma are warranted for further investigation since 1 miRNA can target hundreds of mRNAs and many miRNA-mRNA interactions are still unclear. Therefore, whether the anti$m i R-3162-3 p$ targeting $\beta$-catenin can be used as a new target for asthma treatment needs to be further explored.

\section{Acknowledgements}

This work was supported by the National Natural Science Foundation of China (31670742 and 81671525), the Guangdong Natural Science Foundation (2015A030313521 and
2018A030307043), the Sanming Project of Medicine in Shenzhen (SZSM201512033), Shenzhen Public Service Platform of Molecular Medicine in Pediatric Hematology and Oncology, and the Henan Medical Science and Technology Research Project (2018020355).

\section{Statement of Ethics}

The protocols of the animal experiments were reviewed and approved by the Animal Care and Use Committee in the Laboratory Animal Center of Guangdong Medical University.

\section{Disclosure Statement}

The authors declare no conflicts of interest.

\section{Author Contributions}

X.Z. and Y.H. designed the research; X.Z. wrote the manuscript; J.L., Y.C., F.Z, X.P., X.M., W.L., R.W., B.H., L.M., and Y.B. performed the research; F.Z., X.M., Y.H., and X.Z. analyzed data; and L.M., Y.H., and X.Z. provided advice on experiments. All authors read and approved the final manuscript.

\section{References}

1 Huang H, Lu H, Liang L, Zhi Y, Huo B, Wu L, et al. MicroRNA-744 inhibits proliferation of bronchial epithelial cells by regulating Smad3 pathway via targeting transforming growth factor- $\beta 1$ (TGF- $\beta 1$ ) in severe asthma. Med Sci Monit. 2019;25:2159-68.

2 Chung LP, Paton JY. Fear and courage in children: two sides of the same coin? J Child Fam Stud. 2019;18(4):486-90.

3 Gebert LFR, MacRae IJ. Regulation of microRNA function in animals. Nat Rev Mol Cell Biol. 2019;20(1):21-37.

4 Maneechotesuwan K. Role of microRNA in severe asthma. Respir Investig. 2019;57(1):919.

5 Van den Berge M, Tasena H. Role of microRNAs and exosomes in asthma. Curr Opin Pulm Med. 2019;25(1):87-93.

6 Specjalski K, Jassem E. MicroRNAs: potential biomarkers and targets of therapy in allergic diseases? Arch Immunol Ther Exp. 2019; 67(4):213-23.

7 Wang Y, Yang L, Li P, Huang H, Liu T, He H, et al. Circulating microRNA signatures associated with childhood aAthma. Clin Lab. 2015;61(5-6):467-74.

8 Fang C, Lu W, Li C, Peng X, Wang Y, Huang $\mathrm{X}$, et al. MiR-3162-3p is a novel microRNA that exacerbates asthma by regulating $\beta$-catenin. PLoS One. 2016;11(3):e0149257.
9 Shen J, Zhao J, Ye QY, Gu XD. Interference of miR-943-3p with secreted frizzled-related proteins4 (SFRP4) in an asthma mouse model. Cell Tissue Res. 2019;378(1):67-80.

10 Zhang X, Zhong W, Meng Q, Lin Q, Fang C, Huang X, et al. Ambient PM2.5 exposure exacerbates severity of allergic asthma in previously sensitized mice. J Asthma. 2015; 52(8):785-94.

11 Peng J, Zhang L, Meng Q, Zhang F, Mao X, Liu J, et al. Adverse impact of ambient PM2.5 on expression and trafficking of surfactant protein A through reactive oxygen species damage to lamellar bodies. Toxicol Lett. 2019; 315:47-54.

12 Lu TX, Hartner J, Lim EJ, Fabry V, Mingler MK, Cole ET, et al. MicroRNA-21 limits in vivo immune response-mediated activation of the IL-12/IFN-gamma pathway, Th1 polarization, and the severity of delayed-type hypersensitivity. J Immunol. 2011;187(6):336273.

13 Rebane A, Akdis CA. MicroRNAs in allergy and asthma. Curr Allergy Asthma Rep. 2014; 14(4):424.

14 Martinez-Nunez RT, Bondanese VP, Louafi F, Francisco-Garcia AS, Rupani H, Bedke N, et al. A microRNA network dysregulated in asthma controls IL-6 production in bronchial epithelialcells. PLoSOne. 2014;9(10):e111659.
15 Sharma A, Kumar M, Ahmad T, Mabalirajan U, Aich J, Agrawal A, et al. Antagonism of mmu-mir-106a attenuates asthma features in allergic murine model. J Appl Physiol. 2012; 113(3):459-64

16 Bui H, Amrani Y, Deeney B, Panettieri RA, Tliba O. Airway smooth muscle cells are insensitive to the anti-proliferative effects of corticosteroids: the novel role of insulin growth factor binding protein-1 in asthma. Immunobiology. 2019;224(4):490-6.

17 Wilson RH, Whitehead GS, Nakano H, Free ME, Kolls JK, Cook DN. Allergic sensitization through the airway primes Th17-dependent neutrophilia and airway hyperresponsiveness. Am J Respir Crit Care Med. 2009; 180(8):720-30.

18 Hussain M, Xu C, Lu M, Wu X, Tang L, Wu $\mathrm{X}$. Wnt/ $\beta$-catenin signaling links embryonic lung development and asthmatic airway remodeling. Biochim Biophys Acta. 2017; 1863(12):3226-42.

19 Ogawa H, Azuma M, Tsunematsu T, Morimoto Y, Kondo M, Tezuka T, et al. Neutrophils induce smooth muscle hyperplasia via neutrophil elastase-induced FGF-2 in a mouse model of asthma with mixed inflammation. Clin Exp Allergy. 2018;48(12):171525.

Liu/Chen/Zhang/Peng/Mao/Lu/Wu/ Huang/Bao/Ma/Huang/Zhang 
20 Yao L, Zhao H, Tang H, Song J, Dong H, Zou F, et al. Phosphatidylinositol 3-kinase mediates $\beta$-catenin dysfunction of airway epithelium in a toluene diisocyanate-induced murine asthma model. Toxicol Sci. 2015; 147(1):168-77.

21 Lee H, Bae S, Choi BW, Yoon Y. WNT/ $\beta$ catenin pathway is modulated in asthma patients and LPS-stimulated RAW264.7 macrophage cell line. Immunopharmacol Immunotoxicol. 2012;34(1):56-65.
22 Reuter S, Martin H, Beckert H, Bros M, Montermann E, Belz C, et al. The Wnt/ $\beta$-catenin pathway attenuates experimental allergic airway disease. J Immunol. 2014;193(2):485-95.

23 Dua K, Hansbro NG, Foster PS, Hansbro PM. Targeting microRNAs: promising future therapeutics in the treatment of allergic airway disease. Crit Rev Eukaryot Gene Expr. 2018;28(2):125-7.

24 Svitich OA, Sobolev VV, Gankovskaya LV, Zhigalkina PV, Zverev VV. The role of regulatory RNAs (miRNAs) in asthma. Allergol Immunopathol. 2018;46(2):201-5.
25 Collison A, Mattes J, Plank M, Foster PS. Inhibition of house dust mite-induced allergic airways disease by antagonism of microRNA-145 is comparable to glucocorticoid treatment. J Allergy Clin Immunol. 2011; 128(1):160-7.e4.

26 Yao L, Zhao H, Tang H, Xiong J, Zhao W, Liu $\mathrm{L}$, et al. Blockade of $\beta$-catenin signaling attenuates toluene diisocyanate-induced experimental asthma. Allergy. 2017;72(4):579-89. 